\title{
Some Remarks on the "Null Plane Development" of a Relativistic Quantum Field Theory
}

\author{
S. SCHLIEDER and E. SEILER \\ Max-Planck-Institut für Physik und Astrophysik, München
}

Received October 15, 1971

\begin{abstract}
We give conditions for the existence of field operators on so-called null planes and discuss some consequences of the necessary restriction of the test function space, concerning Haag's theorem and the possibility of unitary mappings intertwining between free fields of different masses. In the last section we discuss conditions under which a unitary representation of the dilatations in the null plane gives rise to a unitary representation of the dilatations in Minkowski space.
\end{abstract}

\section{Introduction}

During the last years there has been a growing interest in the formulation of current algebra and field theory in the so-called "infinite momentum frame". The starting point was the observation made by Fubini and Furlan [1] that certain current algebra sum rules become especially simple if a space component of the momentum (e.g. $p_{z}$ ) goes to infinity. In the meantime several papers treating related problems have been published [2-14]. To express these ideas in a mathematically more rigorous form some authors [8-14] introduced the concept of field operators defined on so-called null planes, i.e. planes tangent to the light cone.

The following remarks are inspired by a talk given by Rohrlich [12] and the paper by Leutwyler, Klauder and Streit [13] (in the following quoted as "LKS"). We hope that they may give some further insight into the questions concerning Haag's theorem, unitary equivalence of different fields and "dilatation invariance" in the case of field operators on null planes.

We use the notation of Leutwyler, Klauder and Streit [13]:

The null plane $\Sigma$ is given by the equation

$$
n_{\mu} x^{\mu}=0
$$

where

$$
n_{\mu}=\frac{1}{\sqrt{2}}(1,0,0,1) .
$$


In the plane $\Sigma$ we use the coordinates

$$
x_{\|}=\frac{1}{\sqrt{2}}\left(x^{0}-x^{3}\right) ; \quad \underline{x}=\left(x^{1}, x^{2}\right),
$$

the coordinate "perpendicular" to $\Sigma$ (i.e. perpendicular with respect to the Euclidean metric in $\mathbb{R}^{4}$ is

$$
x_{\perp}=\frac{1}{\sqrt{2}}\left(x^{0}+x^{3}\right) .
$$

The inner product of two vectors $x, y$ of Minkowski space reads in these coordinates

$$
x \cdot y=x_{\perp} y_{\|}+x_{\|} y_{\perp}-\underline{x} \underline{y} .
$$

For momentum vectors we use the definitions

$$
\begin{aligned}
& p_{\perp}=\frac{1}{\sqrt{2}}\left(p^{0}-p^{3}\right), \\
& p_{\|}=\frac{1}{\sqrt{2}}\left(p^{0}+p^{3}\right), \\
& \underline{p}=\left(p^{1}, p^{2}\right)
\end{aligned}
$$

from which we get

$$
p \cdot x=p_{\perp} x_{\perp}+p_{\|} x_{\|}-\underline{p} \underline{x} .
$$

\section{The Definition of Field Operators on Null Planes}

In this section and in the following ones we will confine the discussion to real scalar fields which satisfy the Wightman axioms. That is to say, besides Lorentz covariance of the fields we assume

(a) the spectral condition for the 4-momentum vector, i.e. the spectrum is contained in $\bar{V}_{+}$(closed forward light cone),

(b) the locality condition for the field operators,

(c) uniqueness of the vacuum state,

(d) temperedness of the operator-valued distributions.

We believe that already this restricted class of field theories is sufficient to make clear the essential features of the problems. It may be useful to treat first the existence problem for field operators on null planes. The discussion of the existence problem leads to some peculiarities which are essential for the following conclusions.

As already stated by LKS in a similar form, for the free field of mass $m$ the transition from

$$
\left\{A_{m}^{0}(h)\right\}=\left\{\int A_{m}^{0}(x) h(x) d^{4} x\right\} ; \quad h \in S\left(\mathbb{R}^{4}\right)
$$


to

$$
\begin{aligned}
\left\{A_{m}^{0}(f)\right\} & =\left\{\int A_{m}^{0}(x) \delta\left(x_{\perp}\right) g\left(x_{\|}, \underline{x}\right) d^{4} x\right\} \\
f(x) & =\delta\left(x_{\perp}\right) g\left(x_{\|}, \underline{x}\right) ; \quad g \in S\left(\mathbb{R}^{3}\right)
\end{aligned}
$$

is not possible for an arbitrary testfunction $g$ of $S\left(\mathbb{R}^{3}\right) . g$ must be chosen from the (closed) linear subspace $\hat{S}\left(\mathbb{R}^{3}\right)$ of $S\left(\mathbb{R}^{3}\right)$ which is defined in the following way:

$$
\hat{S}\left(\mathbb{R}^{3}\right):=\left\{g=\frac{\partial}{\partial x_{\|}} j\left(x_{\|} \cdot \underline{x}\right) ; \quad j \in S\left(\mathbb{R}^{3}\right)\right\}
$$

or through the equivalent condition:

$$
\hat{S}\left(\mathbb{R}^{3}\right):=\left\{g\left(x_{\|}, \underline{x}\right) \in S\left(\mathbb{R}^{3}\right) ; \quad \tilde{g}\left(p_{\|}, \underline{p}\right)=0 \quad \text { for } \quad p_{\|}=0\right\}
$$

(identically in $x$ or $p$, respectively; $\tilde{g}$ is the Fourier transform of $g$ ).

It follows

$$
\left(A_{m}^{0}\left(f_{1}\right) \Omega, A_{m}^{0}\left(f_{2}\right) \Omega\right)=\mathrm{const} \int d^{2} p \Theta\left(p_{\|}\right) \frac{d p_{\|}}{2 p_{\|}} \overline{\tilde{g}}_{1}\left(p_{\|}, p\right) \tilde{g}_{2}\left(p_{\|}, p\right)
$$

with

$$
f_{j}=\delta\left(x_{\perp}\right) g_{j}\left(x_{\|}, \underline{x}\right) \quad \text { and } \quad g_{j} \in \hat{S}\left(\mathbb{R}^{3}\right) ; j=1,2 .
$$

Since the measure $\delta\left(x_{\perp}\right)$ is redundant in the mapping

$$
f \mapsto A_{m}^{0}(f), \quad f=\delta\left(x_{\perp}\right) g\left(x_{\|}, \underline{x}\right) ; \quad g \in \hat{S}\left(\mathbb{R}^{3}\right)
$$

it is possible to define directly the mapping $g \mapsto A_{m}^{0}(g)$ for $g \in \hat{S}\left(\mathbb{R}^{3}\right)$ through the above equations.

For those readers who are interested in the details of this question we give in the appendix an argumentation that it is not possible to extend the positive semidefinite functional

$$
W_{2}(g)=\int \Theta\left(p_{\|}\right) \frac{d p_{\|}}{2 p_{\|}} d^{2} p \tilde{g}\left(p_{\|}, \underline{p}\right)
$$

defined over $\hat{S}\left(\mathbb{R}^{3}\right)$ to a positive semidefinite functional over the whole of $S\left(\mathbb{R}^{3}\right)$ (cf. Gel'fand and Schilow [15], Gel'fand and Wilenkin [16]). Hence we have to remain in the restricted test function space $\hat{S}\left(\mathbb{R}^{3}\right)$, if we do not want to introduce an indefinite metric in state space. (The semidefiniteness of $W_{2}$ means nothing but

$$
\left.W_{2}(g * g)=\left\|A_{m}^{0}(g) \Omega\right\|^{2} \geqq 0 .\right)
$$

The problem of the existence of the field operators on a null-plane for an arbitrary real scalar field $A(x)$ of the Wightman class can be split up into two questions, as in the case of a $t=$ const plane.

Firstly we must have

$$
\|A(g) \Omega\|^{2}<\infty, \quad g \in \hat{S}\left(\mathbb{R}^{3}\right) .
$$


This means

$$
\int_{\tilde{V}_{+}} d \mu(p)\left|\tilde{g}\left(p_{\|}, \underline{p}\right)\right|^{2}<\infty
$$

where $d \mu(p)$ is a positive Lorentz-invariant measure of polynomial increase which is connected with the Lehmann-Källén spectral function through the relation

$$
d \mu(p)=\varrho\left(p^{2}\right) d^{4} p
$$

(of course, $\varrho$ is in general a distribution). Because of

we get

$$
\delta\left(p^{2}-\kappa^{2}\right)=\delta\left(2 p_{\|} p_{\perp}-\underline{p}^{2}-\kappa^{2}\right)
$$

$$
\int_{\bar{V}_{+}} d \mu(p)\left|\tilde{g}\left(p_{\|}, \underline{p}\right)\right|^{2}=\int_{0}^{\infty} \frac{d p_{\|}}{2 p_{\|}} \int_{\mathbb{R}^{2}} d^{2} p\left|\tilde{g}\left(p_{\|}, \underline{p}\right)\right|^{2} \int_{0}^{\infty} \varrho\left(\kappa^{2}\right) d \kappa^{2} .
$$

Thus, for the existence of $A(g) \Omega$ for all $g \in \hat{S}\left(\mathbb{R}^{3}\right)$ we obtain the condition

$$
\int_{0}^{\infty} \varrho\left(\kappa^{2}\right) d \kappa^{2}<\infty
$$

This condition is intermediate between the conditions which must be fulfilled in the case of a plane $t=$ const in order that $A(f) \Omega$ and $\dot{A}(f) \Omega$, respectively, $\left(f \in S\left(\mathbb{R}^{3}\right), \mathbb{R}^{3}\right.$ interpreted as the plane $t=$ const $)$ exist. As is well known these conditions are

$$
\int_{0}^{\infty} \frac{\varrho\left(\kappa^{2}\right)}{\sqrt{1+\kappa^{2}}} d \kappa^{2}<\infty \text { and } \int_{0}^{\infty} \varrho\left(\kappa^{2}\right) \sqrt{1+\kappa^{2}} d \kappa^{2}<\infty
$$

respectively.

The second question concerns the existence of a dense domain for $A(g), g \in \hat{S}\left(\mathbb{R}^{3}\right)$. Here we have to impose conditions on the $n$-pointfunctions or the truncated $n$-point-functions which are similar to those needed for the existence of the field operators on surfaces $t=$ const (essentially we have to require that the increase in momentum space be not stronger than the increase of the two-point-function).

\section{Unitary Mappings of Free Fields; Analogues to Haag's Theorem and the Theorem of Hall and Wightman for Null Planes}

Let $A_{m_{1}}^{0}$ and $A_{m_{2}}^{0}$ be two free fields with masses $m_{1}$ and $m_{2}$, respectively $\left(m_{1} \neq m_{2}\right)$; let $A_{m_{2}}^{0}$ be irreducible in $\mathbb{H}_{1}$ (with vacuum state $\left.\Omega_{1}\right), A_{m_{2}}^{0}$ irreducible in $\mathbb{H}_{2}$ (with vacuum state $\Omega_{2}$ ). LKS discovered that the algebras $\mathfrak{I}_{1}^{0}(\Sigma)$ and $\mathfrak{H}_{2}^{0}(\Sigma)$ generated by

$$
\begin{aligned}
& \left\{A_{m_{1}}^{0}(g), g \in \hat{S}\left(\mathbb{R}^{3}\right)\right\} \\
& \left\{A_{m_{2}}^{0}(g), g \in \hat{S}\left(\mathbb{R}^{3}\right)\right\}, \quad \text { respectively }
\end{aligned}
$$

5 Commun math. Phys., Vol 25 
are irreducible (in fact they are identical with the algebras generated by the field operators smeared with the test functions of $S\left(\mathbb{R}^{4}\right)$ ), and can be mapped unitarily onto each other in the following way: There exists a unitary operator $U$ with the properties

$$
\begin{aligned}
U \Omega_{1} & =\Omega_{2} \\
U A_{m_{1}}^{0}(g) U^{+} & =A_{m_{2}}^{0}(g)
\end{aligned}
$$

for every $g \in \hat{S}\left(\mathbb{R}^{3}\right)$.

This fact is closely connected with a property of the 2-point-functionals: Over $\hat{S}\left(\mathbb{R}^{3}\right)$ the 2-point-functionals of free fields are independent of the mass (compare LKS or the equations of Section 2 of this paper).

If we recall the well-known fact that a corresponding unitary mapping does not exist for operators on a plane $t=$ const, we seem to be confronted with a very remarkable property of field operators on null planes. The reason for the existence of such a unitary mapping, however, lies in the fact that the test function space $\hat{S}\left(\mathbb{R}^{3}\right)$ is too restricted to characterize the local properties of the field operators.

To shed some light on this situation, we shall give another example concerning the same case of the two fields $A_{m_{1}}^{0}$ and $A_{m_{2}}^{0}$ showing how it is possible to map irreducible algebras onto each other if the test function space is chosen adequately.

1. As test function space we use $\hat{S}_{1}\left(\mathbb{R}^{4}\right)$, defined as follows:

$$
\begin{aligned}
& \hat{S}_{1}\left(\mathbb{R}^{4}\right):=\left\{h \in S\left(\mathbb{R}^{4}\right) ; \quad \tilde{h}\left(\boldsymbol{p}, \pm \sqrt{m_{1}^{2}+\boldsymbol{p}^{2}}\right)\right. \\
& \left.=\sqrt[4]{\frac{m_{2}^{2}+\boldsymbol{p}^{2}}{m_{1}^{2}+\boldsymbol{p}^{2}}} \tilde{h}\left(\boldsymbol{p}, \pm \sqrt{m_{2}^{2}+\boldsymbol{p}^{2}}\right)\right\} .
\end{aligned}
$$

The factor $\sqrt[4]{\frac{m_{2}^{2}+\boldsymbol{p}^{2}}{m_{1}^{2}+\boldsymbol{p}^{2}}}$ is chosen as to yield

$$
\int \frac{d^{3} p}{\sqrt{m_{1}^{2}+\boldsymbol{p}^{2}}}\left|\tilde{h}\left(\boldsymbol{p}, \sqrt{m_{1}^{2}+\boldsymbol{p}^{2}}\right)\right|^{2}=\int \frac{d^{3} p}{\sqrt{m_{2}^{2}+\boldsymbol{p}^{2}}}\left|\tilde{h}\left(\boldsymbol{p}, \sqrt{m_{2}^{2}+\boldsymbol{p}^{2}}\right)\right|^{2}
$$

and hence

$$
\left\|A_{m_{1}}^{0}(h) \Omega_{1}\right\|^{2}=\left\|A_{m_{2}}^{0}(h) \Omega_{2}\right\|^{2} .
$$

This enables us to find a unitary operator $U$ fulfilling

$$
U \Omega_{1}=\Omega_{2} ; \quad U A_{m_{1}}^{0}(h) U^{+}=A_{m_{2}}^{0}(h)
$$

for every $h \in \hat{S}_{1}\left(\mathbb{R}^{4}\right)$.

The fields $A_{m_{1}}^{0}$ and $A_{m_{2}}^{0}$ over $\hat{S}_{1}\left(\mathbb{R}^{4}\right)$ generate an irreducible algebra, as is immediately seen from the following observation: Let $f$ be the Fourier transform of a function $f \in S\left(\mathbb{R}^{4}\right)$. There exist functions $h_{j} \in \hat{S}_{1}\left(\mathbb{R}^{4}\right)$ with

$$
\tilde{h}_{j}\left(\boldsymbol{p}, \pm \sqrt{m_{j}^{2}+\boldsymbol{p}^{2}}\right)=\tilde{f}\left(\boldsymbol{p}, \pm \sqrt{m_{j}^{2}+\boldsymbol{p}^{2}}\right)
$$


and this yields

$$
A_{m_{j}}^{0}(f)=A_{m_{j}}^{0}(h) ; \quad j=1,2 .
$$

We can express this fact in a different fashion: at first there are two mappings from $\hat{S}_{1}\left(\mathbb{R}^{4}\right)$ into the set of operators of the Hilbert spaces $\mathbb{H}_{1}$ and $\mathbb{H}_{2}$, respectively:

$$
\begin{aligned}
& \hat{\Phi}_{1}: h \mapsto A_{m_{1}}^{0}(h) \\
& \hat{\Phi}_{2}: h \mapsto A_{m_{2}}^{0}(h)
\end{aligned} \quad\left(h \in \hat{S}_{1}\left(\mathbb{R}^{4}\right)\right) .
$$

These two mappings are unitarily equivalent in the following sense: there exists a unitary mapping

$$
U: \mathbb{H}_{1} \rightarrow \mathbb{H}_{2}
$$

which induces an isomorphism $\tau_{u}$ of the field algebra $\mathfrak{A}_{1}$ (generated by the field $A_{m_{1}}^{0}$ ) into the field algebra $\mathfrak{A}_{2}$ (generated by the field $A_{m_{2}}^{0}$ )

$$
\tau_{u}: \mathfrak{U}_{1} \ni A_{1} \mapsto U A_{1} U^{-1} \in \mathfrak{U}_{2}
$$

so that the following diagram is commutative:

(that means

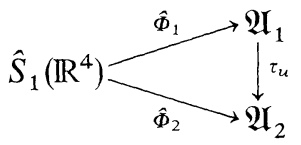

or equivalently

$$
\hat{\Phi}_{2}=\tau_{u} \circ \hat{\Phi}_{1}
$$

$$
\left.\hat{\Phi}_{1}=\tau_{u}^{-1} \circ \hat{\Phi}_{2}\right) \text {. }
$$

If we take into account the field equations

$$
\begin{aligned}
& \left(\square+m_{1}^{2}\right) A_{m_{1}}^{0}(x)=0, \\
& \left(\square+m_{2}^{2}\right) A_{m_{2}}^{0}(x)=0,
\end{aligned}
$$

we see that the continuations of the mappings $\hat{\Phi}_{1}, \hat{\Phi}_{2}$ from $\hat{S}_{1}\left(\mathbb{R}^{4}\right)$ to $S\left(\mathbb{R}^{4}\right)$ have to be constructed in the following way: We divide $S\left(\mathbb{R}^{4}\right)$ into equivalence classes $K_{j}$ and $L_{j}$, respectively, by the equivalence relations

$$
\begin{array}{lll}
f_{1} \sim_{K} f_{2} & \text { iff } & \left.\tilde{f}_{1}(p)\right|_{p^{2}=m_{1}^{2}}=\left.\tilde{f}_{2}(p)\right|_{p^{2}=m_{1}^{2}}, \\
f_{1} \sim_{L} f_{2} & \text { iff } & \left.\tilde{f}_{1}(p)\right|_{p^{2}=m_{2}^{2}}=\left.\tilde{f}_{2}(p)\right|_{p^{2}=m_{2}^{2}} .
\end{array}
$$

Each class $K_{j}$ and $L_{j}$ contains functions of $\hat{S}_{1}\left(\mathbb{R}^{4}\right)$; the continuation of $\hat{\Phi}$ is now made in the following way: each $f \in K_{j}$ is mapped on $A_{m_{1}}^{0}\left(h_{j}\right)$ where $h_{j} \in K_{j} \cap \hat{S}_{1}\left(\mathbb{R}^{4}\right) ; \hat{\Phi}_{2}$ is continued analogously. (This determines the continuation uniquely.) Thus we have defined two mappings from $S\left(\mathbb{R}^{4}\right)$ into the set of operators in $\mathbb{H}_{1}$ and $\mathbb{H}_{2}$

$$
\Phi_{1}: S\left(\mathbb{R}^{4}\right) \ni f \mapsto A_{m_{1}}^{0}(f):=A_{m_{1}}^{0}(h)
$$


where $f \sim_{K} h$,

where $f \sim{ }_{L} h$.

$$
\Phi_{2}: S\left(\mathbb{R}^{4}\right) \ni f \mapsto A_{m_{2}}^{0}(f):=A_{m_{2}}^{0}(h)
$$

There is, however, no unitary operator from $\mathbb{H}_{1}$ to $\mathbb{H}_{2}$ which makes a diagram corresponding to (3.14) (with $\hat{\Phi}_{1}, \hat{\Phi}_{2}$ replaced by $\Phi_{1}, \Phi_{2}$ ) commutative, i.e. which fulfils $U A_{m_{1}}^{0}(f) U^{+}=A_{m_{2}}^{0}(f)$ for every $f \in S\left(\mathbb{R}^{4}\right)$.

The common feature of both examples is this: The mappings from the test function spaces $\hat{S}\left(\mathbb{R}^{3}\right), \hat{S}_{1}\left(\mathbb{R}^{4}\right)$, into the set of Hilbert space operators do not permit characterization of the local properties of the operator-valued distributions, unless the field equations are used to extend these mappings to the whole of $S\left(\mathbb{R}^{4}\right)$.

So far we described the situation arising if we define operators on null planes. Some other features become evident, if we discuss the Wightman functions on a null plane $\Sigma$ and their analytic continuations into Minkowski space.

It is clear that under the assumptions made at the beginning of this section

$$
\begin{aligned}
& \left(\Omega, A\left(x_{\|}^{1}, x_{\perp}, \underline{x}^{1}\right) \ldots A\left(x_{\|}^{n}, x_{\perp}, \underline{x}^{n}\right) \Omega\right) \\
& =\left.W_{n}\left(\xi_{1}, \ldots, \xi_{n-1}\right)\right|_{\left(\xi_{1}\right)_{\perp}=\cdots=\left(\xi_{n-1}\right)_{\perp}=0}
\end{aligned}
$$

is analytic for $x_{\|}^{i} \neq x_{\|}^{j}(i, j=1, \ldots, n)$.

Stated in this way, the situation looks very different; we shall contrast it with the corresponding one for $t=$ const:

Theorem of Hall and Wightman for Planes $t=$ const:

$$
W_{2}(\xi), \quad W_{3}\left(\xi_{1}, \xi_{2}\right), \quad W_{4}\left(\xi_{1}, \xi_{2}, \xi_{3}\right)
$$

are uniquely determined by their values for $\xi^{0}=0, \xi_{1}^{0}=\xi_{2}^{0}=0$ and $\xi_{1}^{0}=\xi_{2}^{0}=\xi_{3}^{0}=0$, respectively.

\section{Analogue to the Theorem of Hall and Wightman for Null Planes:}

$W_{2}(\xi)$ and $W_{3}\left(\xi_{1}, \xi_{2}\right)$ are uniquely determined by their values for $\xi_{\perp}=0$ and $\left(\xi_{1}\right)_{\perp}=\left(\xi_{2}\right)_{\perp}=0$, respectively.

The proof is elementary and closely follows the proof of the theorem of Hall and Wightman as given in the book by Streater and Wightman [17]:

$W_{2}$ and $W_{3}$ have to be Lorentz-invariant. Thus, if we know $W_{1}$ and $W_{2}$ for $\xi_{\perp}=0$ and $\left(\xi_{1}\right)_{\perp}=\left(\xi_{2}\right)_{\perp}=0$, respectively, we also know them for every point which can be reached from these points by a Lorentztransformation. If the points, for which $W_{2}$,nd $W_{3}$ are thus determined, contain an open set of $\mathbb{R}^{4}$ and $\mathbb{R}^{4} \times \mathbb{R}^{4}$, respectively, $W_{2}$ and $W_{3}$ are determined everywhere by analytic continuation.

Let $\xi_{0}$ be a spacelike vector with $\left(\xi_{0}\right)_{\perp}=0$ (i.e. $\xi_{0} \in \Sigma$ ). By a Lorentztransformation $\xi_{0}$ can be transformed into any vector $\xi$ with $\xi^{2}=\xi_{0}^{2}$. 
For $\lambda \in \mathbb{R}, \lambda \xi_{0}$ is an element of $\Sigma$. From the vectors of the form $\lambda \xi_{0}$ we can reach every spacelike vector by a Lorentz-transformation; the set of all spacelike vectors is an open set of $\mathbb{R}^{4}$.

Now let $\xi_{1}, \xi_{2}$ be two spacelike vectors which span a spacelike plane $^{1}$, i.e. which fulfil

$$
\left|\begin{array}{cc}
\xi_{1}^{2} & \xi_{1} \cdot \xi_{2} \\
\xi_{1} \cdot \xi_{2} & \xi_{2}^{2}
\end{array}\right|<0
$$

By choosing a lightlike vector $\xi_{3}$ we can form a triple of vectors which spans a hyperplane $E$ tangent to the light cone (i.e. a null plane). Every hyperplane of this kind can be transformed into $\Sigma$ by a Lorentz-transformation (because such a hyperplane is characterized by the lightlike "normal vector" $\xi_{3}$; if we apply a Lorentz-transformation which maps $\xi_{3}$ onto $n_{\mu}=\frac{1}{\sqrt{2}}(1,0,0,1)$, it maps $E$ onto $\left.\Sigma\right)$. Thus every pair of spacelike vectors which fulfils (3.24) can be mapped by a Lorentz-transformation onto a pair of vectors lying in $\Sigma$, or, stated differently, every pair of spacelike vectors fulfilling (3.24) can be reached from a pair of vectors lying in $\Sigma$ by a Lorentz-transformation. Clearly the set of pairs of spacelike vectors fulfilling (3.24) is an open set of $\mathbb{R}^{8}=\mathbb{R}^{4} \times \mathbb{R}^{4}$. This proves our statement.

Remark. It is not possible to prove a similar statement for the fourpoint-function: Every triple of vectors $\xi_{1}, \xi_{2}, \xi_{3} \in \Sigma$ fulfils

$$
D\left(\xi_{1}, \xi_{2}, \xi_{3}\right):=\left|\begin{array}{ccc}
\xi_{1}^{2} & \xi_{1} \cdot \xi_{2} & \xi_{1} \cdot \xi_{3} \\
\xi_{1} \cdot \xi_{2} & \xi_{2}^{2} & \xi_{2} \cdot \xi_{3} \\
\xi_{1} \cdot \xi_{3} & \xi_{2} \cdot \xi_{3} & \xi_{3}^{2}
\end{array}\right|=0 .
$$

$D$ is an entire function on $\mathbb{C}^{12}$. It vanishes, when restricted to $\Sigma$, but clearly it does not vanish identically. This shows that the values on $\Sigma$ are not sufficient to determine an analytic function of three four-vectors.

So we have the following results:

If we try to define field operators on a null plane, the space of test functions even in the case of a free field must be so restricted that we cannot characterize the local properties of the field (nor the rest mass of a free field). If, on the other hand, we consider the Wightman functionals with arguments on the null-plane $\Sigma$, the values of the 2- or 3-pointfunctions on this plane determine the values of the 2- and 3-pointfunctions, respectively, for arbitrary arguments by analytic continuation. This property of the 2-point-function shows that the analogue of Haag's theorem is still true if one uses the Wightman functions instead of the field operators themselves.

\footnotetext{
${ }^{1}$ This implies that $\left(\xi_{1}, \xi_{2}\right)$ is a Jost point.
} 


\section{Dilatation Invariance}

We intend to make a comment on the meaning of "dilatation invariance on null planes" (cf. Rohrlich and Streit [14]), which by some people has been looked upon as a possibility of giving a more precise meaning to the intuitive concept of "dilatation invariance on the light cone" (cf. [18-22]).

When speaking of space-time symmetries we usually think of a group of transformations of Minkowski space $M_{4}$ (or a unitary representation of that group in state space). In the framework of relativistic local quantum field theory only those transformations make sense under which the notions "spacelike", "timelike", and "lightlike" are invariant. This restricts the admissible transformations to the group $G$ which is generated by inhomogeneous Lorentz-transformations and dilatations, as has been shown by Zeeman [23]. Of course it is also possible to consider mappings of submanifolds of $M_{4}$ (e.g. $\Sigma$ ) onto themselves. If we perform, for instance, a dilatation of the plane $x_{\perp}=\tau$ this can be interpreted (at least in the free field case) as a dilatation of the wave packets at the "time" $\tau$; whether this corresponds to a dilatation also at a different "time" $\tau$ depends on the "Hamiltonian" $P_{\perp}$. In general this will not be the case; that is to say, in general such dilatations of null planes will not correspond to dilatations of $M_{4}$ (they cannot correspond to any other transformation of $G$, as the only elements of $G$ which induce dilatations on $\Sigma$ are dilatations of $M_{4}$ ).

It can be shown that in fact the following general statement is true:

Let $A$ be a real scalar field which makes sense as a distribution over $\hat{S}(\Sigma)$. Assume that the dilatation group of $\Sigma$ is unitarily implementable, i.e. that there is a group of unitary operators $U(\lambda)(\lambda>0)$ which fulfil

(where

$$
U(\lambda) A(g) U^{+}(\lambda)=\lambda^{d} A\left(g_{\lambda}\right)
$$

$$
\left.g_{\lambda}\left(x_{\|}, \underline{x}\right)=\lambda^{-3} g\left(\lambda^{-1} x_{\|}, \lambda^{-1} \underline{x}\right)\right)
$$

for all $g \in \hat{S}(\Sigma)$ and

$$
U(\lambda) \Omega=\Omega
$$

Then these operators $U(\lambda)$ can be interpreted as representing the group of dilatations of $M_{4}$ if and only if $A$ is a free field of mass zero.

Proof. If we want to interprete $U(\lambda)$ as representing the dilatation $x \mapsto \lambda x\left(x \in M_{4}\right)$ we must have for every $f \in S\left(\mathbb{R}^{4}\right)$

where

$$
U(\lambda) A(f) U^{+}(\lambda)=\lambda^{d} A\left(f_{\lambda}\right)
$$

$$
f_{\lambda}(x)=\lambda^{-4} f\left(\lambda^{-1} x\right) .
$$


This implies (together with (4.3)) that the two-point-function has the form

$$
W_{2}(x)=\operatorname{const} \int \Theta\left(p_{0}\right)\left(p^{2}\right)_{+}^{d-2} e^{i p x} d^{4} p
$$

for $d>1$ or

$$
W_{2}(x)=\text { const } \int \Theta\left(p_{0}\right) \delta\left(p^{2}\right) e^{i p x} d^{4} p
$$

for $d=1$ (cf. Gel'fand and Schilow [15]; the case $d<1$ is not possible because of the positive-definiteness of $W_{2}$ ). But the assumption that the restriction of the field $A$ to the plane $\Sigma$ makes sense excludes $d>1$ (cf. Section 2). So we are left with $d=1$; it is well known that (4.5) implies that $A$ is a free field of mass zero (cf. Pohlmeyer [24]).

Acknowledgements. We wish to thank J. R. Klauder and L. Streit who helped us to avoid some errors, and R. Haag who gave us hints for a more clear and concise presentation.

\section{Appendix}

Every positive semidefinite functional over $S\left(\mathbb{R}^{3}\right)$ is the Fourier transform of a positive tempered measure (Gel'fand and Wilenkin [16]); $\frac{1}{p_{\|}} \Theta\left(p_{\|}\right)$however, is not a measure on $\mathbb{R}^{3}$.

More explicitly we can see that every extension of $W_{2}$ on the whole of $S\left(\mathbb{R}^{3}\right)$ necessarily violates the positivity condition: We can decompose $S\left(\mathbb{R}^{3}\right)$ in the following way into a direct sum:

$$
S\left(\mathbb{R}^{3}\right)=\hat{S}\left(\mathbb{R}^{3}\right) \oplus\left\{f_{0}\right\} \otimes S\left(\mathbb{R}^{2}\right)
$$

where $\left\{f_{0}\right\}$ is the one-dimensional space spanned by a function $f_{0}\left(x_{\|}\right)$ $\in S(\mathbb{R})$ with $\tilde{f}_{0}(0) \neq 0$; for the sake of definiteness let us choose $\tilde{f}_{0}\left(p_{\|}\right)$ $=e^{-\frac{1}{2} p p^{2}}$. This corresponds to the decomposition

$$
S\left(\mathbb{R}^{3}\right) \ni \tilde{\varphi}\left(p_{\|}, \underline{p}\right)=\left[\tilde{\varphi}\left(p_{\|}, \underline{p}\right)-f_{0}\left(p_{\|}\right) \tilde{\varphi}(0, \underline{p})\right]+\tilde{f}_{0}\left(p_{\|}\right) \tilde{\varphi}(0, \underline{p}) .
$$

Every continuation of the functional $W_{2}$ which is already defined on $\hat{S}\left(\mathbb{R}^{3}\right)$ thus is of the form

$$
W_{2}(\varphi)=\int \frac{d p_{\|}}{2 p_{\|}} \Theta\left(p_{\|}\right) d^{2} p\left[\tilde{\varphi}\left(p_{\|}, p\right)-\tilde{f}_{0}\left(p_{\|}\right) \tilde{\varphi}(0, p)\right]+T\left(\tilde{\varphi}_{r}\right)
$$

where $\tilde{\varphi}_{r}(\underline{p})=\tilde{\varphi}(0, \underline{p})$ and $T \in S^{\prime}\left(\mathbb{R}^{2}\right)$.

Every extension of this form is automatically continuous, since $\hat{S}\left(\mathbb{R}^{3}\right)$ and $\left\{f_{0}\right\} \otimes S\left(\mathbb{R}^{2}\right)$ are closed subspaces of $S\left(\mathbb{R}^{3}\right)$. A different choice of $f_{0}$ is equivalent to a different choice of $T$.

Now let us consider the sequence

$$
\tilde{\varphi}_{n}\left(p_{\|}, \underline{p}\right)=e^{-\frac{1}{2} n p_{\|}^{2}-\frac{1}{2} \underline{p}^{2}}
$$


for which we get

$$
W_{2}\left(\varphi_{n}\right)=\frac{1}{2 \pi} \frac{d p_{\|}}{2 p_{\|}} \Theta\left(p_{\|}\right)\left(e^{-\frac{1}{2} n p_{\|}^{2}}-e^{-\frac{1}{2} p_{\|}^{2}}\right)+T\left(e^{-\frac{1}{2} p^{2}}\right) .
$$

As is immediately seen, $W_{2}\left(\varphi_{n}\right)$ becomes negative for sufficiently high $n$, although $\tilde{\varphi}_{n} \geqq 0$ for every $n$; this fact does not depend on the choice of $T \in S\left(\mathbb{R}^{2}\right)$. This shows that $W_{2}$ cannot be extended to a positive semidefinite distribution over $S\left(\mathbb{R}^{3}\right)$.

\section{References}

1. Fubini, S., Furlan, G.: Physics 1, 229 (1965).

2. Weinberg, S.: Phys. Rev. 150, 1313 (1966).

3. Bardakci, K., Segrè, G.: Phys. Rev. 159, 1263 (1967).

4. Susskind, L.: Phys. Rev. 165, 1535 (1967). Jersak, J., Stern, J.: Dubna preprints E2-3469 (191,?) and E2-3990 (1968).

6. Bardakci, K., Halpern, M. B.: UCRL preprint 18360 (1968).

7. Garwin, C.: UCRL preprint 19356 (1969).

8. Leutwyler, H.: Acta Phys. Austr. Suppl. V, 320 (1968), Springer Tracts Mod. Phys. 50, 29. Berlin-Heidelberg-New York: Springer 1969.

9. Kogut, J. B., Soper, D.E.: Phys. Rev. D 1, 2901 (1970).

10. Bjorken, J.D., Kogut, J.B., Soper, D. E.: Phys. Rev. D3, 1382 (1971).

11. Neville, R. A., Rohrlich,F.: Phys. Rev. D3, 1692 (1971).

12. Rohrlich, F.: Lecture given at the X. Internationale Universitätswochen, Schladming 1971.

13. Leutwyler, H., Klauder, J.R., Streit, L.: Nuovo Cimento 66 A, 536 (1970).

14. Rohrlich, F., Streit, L.: Syracuse University preprint (1971).

15. Gel'fand, I. M., Schilow, G. E.: Verallgemeinerte Funktionen I. Berlin: VEB Deutscher Verlag der Wissenschaften 1967.

16. - Wilenkin, N.J.: Verallgemeinerte Funktionen IV. Berlin: VEB Deutscher Verlag der Wissenschaften 1964.

17. Streater, R.F., Wightman, A.S.: PCT, spin and statistics and all that. New York: W. A. Benjamin Inc. 1964.

18. Mitter, H.: Nuovo Cimento 32, 1789 (1964).

19. Frishman, Y.: Phys. Rev. Letters 25, 966 (1970).

20. Brandt, R. A., Preparata, G.: CERN preprint TH 1208.

21. Fritzsch, H., Gell-Mann, M.: Proc. of the Int. Conf. on Fundamental Interactions, Coral Gables 1971.

22. Leutwyler, H., Stern, J.: Nucl. Phys. B 20, 77 (1970).

23. Zeeman, E. C.: J. Math. Phys. 5, 490 (1964).

24. Pohlmeyer, K.: Commun. math. Phys. 12, 204 (1969).

S. Schlieder

E. Seiler

MPI f. Physik und Astrophysik

D-8000 München 23, Föhringer Ring 6 Germany 